\title{
Editorial
}

\section{Abordar la violencia contra las mujeres desde una perspectiva integral}

\author{
Cristina Vélez Valencia MsC, BA, PhD(c)', \\ Laura Palacios Astorquiza MSc, BA ${ }^{2}$
}

En los últimos veinte años, la violencia contra las mujeres ha estado presente en la agenda pública nacional e internacional. Los tratados internacionales, los desarrollos normativos locales y nacionales y el desarrollo de políticas públicas que tienen como centro una vida libre de violencia para las mujeres son ejemplo de estos avances ${ }^{3}(1-4)$. En estas décadas de trabajo se ha identificado la importancia de abordar este problema de forma integral. ¿Por qué? Porque las causas de la violencia contra la mujer son multifactoriales, las afectaciones trascienden la esfera física del golpe y porque la atención debe ser interdisciplinaria. Así mismo, las consecuencias de este tipo de violencia afectan a las mujeres en múltiples esferas.

En Colombia, la Ley 1257 de 2008 reconoce la importancia de llevar a cabo acciones coordinadas entre las distintas entidades que hacen parte de los procesos de información, prevención, orientación, protección, sanción, reparación y estabilización de los derechos de las mujeres víctimas de violencia. Asimismo, establece medidas específicas en el ámbito laboral, familiar y de la salud.

1 Secretaria Distrital de la Mujer, Alcaldía Mayor de Bogotá.

2 Secretaría Distrital de la Mujer.

3 En el marco jurídico internacional se encuentran la Convención sobre la eliminación de todas las formas de discriminación contra la mujer (CEDAW), aprobada en 1979 por la por la Asamblea General de las Naciones Unidas y ratificada por Colombia en 2007. La Convención define la discriminación contra las mujeres y establece la noción de igualdad sustantiva, amplía las responsabilidades por discriminación a terceros, obliga a los estados a la adopción de medidas concretas y reconoce el papel de la cultura en la discriminación contra las mujeres (1). En 1993, la Declaración de Viena sobre derechos humanos, emanada de la Conferencia de Viena, reconoce los derechos de las mujeres como Derechos Humanos (2). Al año siguiente, se crea la Convención Interamericana para prevenir, sancionar y erradicar la violencia contra la mujer (Convención de Belém Do Pará), que trata específicamente la violencia contra las mujeres como una violación a los derechos humanos. La convención también reconoce este tipo de violencia como una manifestación de relaciones históricas de desigualdad entre hombres y mujeres (3). En 1995, se promulga la Declaración y Plataforma de Acción de Beijing, la cual reitera que los derechos de las mujeres son derechos humanos. También formula compromisos en 12 áreas (entre ellas, pobreza, educación, economía y medio ambiente), reconociendo que es necesario lograr avances en todas para materializar efectivamente los derechos de las mujeres (4). En el marco jurídico nacional, en la Constitución Política de Colombia se encuentran el Artículo 13, acerca de la igualdad real y efectiva de todas las personas ante la Ley, Artículo 42, acerca de la familia y la igualdad de derechos de la pareja y el Artículo 43, sobre la igualdad de derechos y oportunidades para hombres y mujeres. Con respecto a violencia contra las mujeres, se debe tener en cuenta la Ley 1257 de 2008, "Por la cual se dictan normas de sensibilización, prevención y sanción de formas de violencia y discriminación contra las mujeres, se reforman los Códigos Penal, de Procedimiento Penal, la Ley 294 de 1996 y se dictan otras disposiciones", la cual define la violencia contra las mujeres de manera amplia, incluyendo la violencia económica y patrimonial. La ley 1719 de 2014, "Por la cual se modifican algunos artículos de las Leyes 599 de 2000, 906 de 2004 y se adoptan medidas para garantizar el acceso a la justicia de las víctimas de violencia sexual, en especial la violencia sexual con ocasión del conflicto armado, y se dictan otras disposiciones", establece medidas respecto a la violencia sexual, dentro y fuera del conflicto armado. Finalmente, La Ley 1761 de 2015, "Por la cual se crea el tipo penal de feminicidio como delito autónomo y se dictan otras disposiciones", tipifica el delito de feminicidio. 
De acuerdo con esto, una visión integral de la violencia contra las mujeres debe incluir un análisis de factores educativos, económicos, culturales, políticos y de salud. Un ejemplo de este abordaje integral es el análisis que desarrolla Julieta Lemaitre sobre la incidencia de los títulos de propiedad de las viviendas sobre la violencia, cuando estos son compartidos entre hombres y mujeres o están en nombre de mujeres, sin importar el nivel de ingresos de la familia, hay menos violencia (5). Asimismo, Frieddeman estudió la incidencia de la alfabetiación judicial de las mujeres en la reducción de violencias y observó que las mujeres que conocen sus derechos y que están empoderadas políticamente tienen menos riesgo de ser víctimas de violencias (6).

En otros contextos, también se han investigado factores como el empoderamiento económico y su efecto en la ocurrencia de hechos de violencia contra las mujeres, donde el rol de estas en la toma de decisiones económicas dentro del hogar se estudia como un factor que incide en el riesgo de sufrir violencias (7). Otro ejemplo es el estudio de la educación que recibe el personal de salud con respecto a la identificación, atención y reporte de casos de violencia contra las mujeres. Se piensa que incluir información acerca de cómo identificar las violencias, sus causas y consecuencias dentro del currículum de carreras afines a la salud puede ser una forma de mejorar la atención y prevención de casos de violencias (8).

Se han realizados estudios de carácter cuantitativo y cualitativo, lo que ha aportado a la comprensión del problema desde distintas perspectivas. Esta edición de Ciencias de la salud contiene dos artículos que contribuyen a esta necesaria mirada integral desde el campo de la salud. El primero es "Violencia contra la mujer: levantamiento de casos registrados en una Comisaría del Estado de Minas Gerais" de Caetano et al., donde las autoras realizan una investigación para recoger estadísticas descriptivas con el fin de caracterizar a las víctimas de violencia contra la mujer y a sus agresores (9). El estudio arroja un perfil de mujeres víctimas de violencia jóvenes y adultas entre 20 y 49 años, hombres victimarios en el mismo rango de edad y en su mayoría cónyuges o excónyuges de las víctimas.

Los hallazgos de esta investigación concuerdan, en gran parte, con las cifras que se conocen para la ciudad de Bogotá, en cuanto a los perfiles de las víctimas, el sexo de los agresores y los tipos de violencias ocurridas. Sin embargo, los datos no concuerdan en cuanto a diferencias en la ocurrencia de hechos violentos contra las mujeres, según el día de la semana. Si bien, las autoras no encuentran consistencia en los días de la semana en los que más ocurren hechos de violencia, en Bogotá los domingos son el día en el que más se reportan casos de violencia de pareja ${ }^{4}$. Este comportamiento sugiere que el consumo de alcohol los fines de semana incide en estos hechos violentos, consistente con lo que señalan Caetano et al. en su estudio de una Comisaría de Familia en minas de Gerais, en el que encontraron que el $25 \%$ de los agresores estaban alcoholizados en el momento de la agresión. Conocer la influencia de factores como el consumo de alcohol es de utilidad al momento de plantear un acercamiento multidimensional al fenómeno, por ejemplo, es posible que una campaña de reducción del consumo de alcohol y de sustancias psicoactivas sea la mejor forma de luchar contra la violencia al interior de la familia.

4 Información preliminar de las cifras correspondientes al año 2016, según el Instituto Nacional de Medicina Legal. Cálculos elaborados por el Observatorio de Mujer y Equidad de Género de la Secretaría Distrital de la Mujer (10). 
En el $45 \%$ de todos los casos de violencia física contra mujeres en Bogotá, incluyendo muerte violenta en manos de un tercero, violencia de pareja, violencia sexual y violencia al interior de la familia, el agresor fue la pareja o la expareja de la mujer. A pesar de este dato contundente, todavía falta mucha sistematicidad a la hora de perfilar quiénes son los perpetuadores de esta forma de violencia (10). Contar con perfiles tanto de víctimas como de victimarios es sumamente necesario para poder diseñar políticas efectivas de prevención y atención a las violencias y los datos recogidos en el estudio en Minas Gerais son valiosos como una primera exploración. La información que arroja este tipo de estudios puede ser complementada con datos cualitativos que ayuden a explicar las implicaciones de los resultados y con análisis estadísticos que trasciendan lo descriptivo. Adicionalmente, la inclusión de preguntas que se refieran a otro tipo de factores que puedan incidir en las situaciones de violencia puede ser pertinente. Por ejemplo, en este estudio hubiera sido interesante preguntarles a las mujeres acerca de su situación de vivienda, independencia económica o empoderamiento político, siguiendo los ejemplos mencionados al principio de este editorial. En el diseño de políticas de prevención, persiste la deuda de identificar patrones que nos ayuden a reconocer e intervenir de manera más efectiva potenciales casos de violencias contra las mujeres.

El segundo artículo sobre el tema en esta edición es de corte cualitativo. Posada, Mendoza, Orozco, et al. exploran cómo los roles de género de las mujeres desplazadas en Medellín influyen en la percepción que ellas tienen de su situación de salud (11). Las investigadoras utilizan la teoría fundamentada y concluyen que los resultados sugieren una relación entre el desplazamiento, el cambio de roles de género y la percepción negativa de las mujeres frente a su salud mental y física.

Entre otros factores, las autoras indagan acerca de cómo, al ocupar el rol de proveedoras económicas de la familia, las mujeres deben asumir una doble labor al ser también las encargadas de las labores de cuidado del hogar y de los hijos. Esto les genera a las mujeres una percepción negativa respecto a su propia salud física y mental. Sobre este punto, se considera valioso el partir de una concepción del género como un determinante en la salud y en la forma en la que las mujeres viven y conciben la enfermedad. Esta investigación es un punto de partida para preguntarse cómo explorar a profundidad estas relaciones entre los roles de género, las percepciones de salud y enfermedad y el acercamiento a la oferta estatal en servicios de salud por parte de las mujeres. Una mejor comprensión de la forma en la que estos roles afectan asuntos como la adherencia y la selección de servicios podría derivar en herramientas valiosas para la formulación de políticas de salud pública.

De forma similar, el texto hace evidente la necesidad de políticas de salud pública que se enmarquen en intervenciones integrales, especialmente en lo que se refiere a las políticas de la economía del cuidado. Mientras las mujeres continúen siendo mayoritariamente responsables de las labores de cuidado, su autonomía seguirá siendo limitada, afectando no solo su salud — concebida como bienestar en un sentido amplio y no solo ausencia de enfermedad — sino también su situación de vulnerabilidad frente a distintos tipos de violencia. De acuerdo con esto, el diseño de políticas se enfrenta al reto de tener en cuenta las labores tradicionalmente llevadas a cabo por las mujeres sin naturalizar los roles de género que limitan su desarrollo por fuera de la esfera doméstica.

Tanto este estudio como el llevado a cabo por Caetano et al. dan muestra de los grandes aportes que brinda la investigación en el campo de la salud respecto al tema de las violencias. Es importante 
resaltar que el estudio de la violencia contra las mujeres como un tema de salud pública implica reconocerlo como un problema que afecta a la sociedad en su conjunto. También, implica que este fenómeno debe ser tratado en los debates de políticas públicas en salud y que requiere un abordaje sistemático por parte del Estado. Además de esto, creemos que la formulación de preguntas de investigación y los análisis que se realicen desde las ciencias médicas pueden aportar al estudio del problema desde perspectivas novedosas para las ciencias sociales y viceversa.

$\mathrm{Al}$ establecer puentes entre diferentes campos de estudio, se pueden tener en cuenta las múltiples causas y factores que inciden en la ocurrencia de los hechos de violencia. Igualmente, se pueden evidenciar las múltiples afectaciones que conllevan las violencias contra las mujeres, no solo en cuanto a la salud sino también en los ámbitos económico, educativo, político y social. Al hacer un acercamiento al problema desde múltiples ángulos y plantear discusiones entre las distintas disciplinas, se podrá contar con una visión multidimensional del fenómeno. De esta forma, estaremos más cerca de consolidar herramientas verdaderamente integrales de prevención y atención a las violencias contra las mujeres.

\section{Referencias}

1. Naciones Unidas. Convención sobre la eliminación de todas las formas de discriminación contra la mujer. 1979. Disponible en: http://www.un.org/womenwatch/daw/cedaw/text/sconvention.htm

2. Naciones Unidas. Declaración y programa de acción de Viena. 1993. Disponible en: http://www.ohchr. org/Documents/Events/OHCHR20/VDPA_booklet_Spanish.pdf

3. Departamento de Derecho Internacional, DEA. Convención interamericana para prevenir, sancionar y erradicar la violencia contra la mujer "Convención de Belem do Para". 1994. Disponible en: http:// www.oas.org/juridico/spanish/tratados/a-61.html

4. Naciones Unidas. Declaración y Plataforma de Acción de Beijing. 1995. Disponible en: http://beijing20. unwomen.org/ /media/headquarters/attachments/sections/csw/bpa_s_final_web.pdf

5. Lemaitre-Ripoll J, García-Jaramillo S, Ramírez-Rodríguez H. Vivienda/violencia: intersecciones de la vivienda y la violencia intrafamiliar en Ciudad Bolívar, Bogotá. Revista de Estudios Sociales. 2014;(50):71-86.

6. Frieddeman-Sáchez G. Legal Literacy and Immovable Property: Laws, Norms and Practices in Colombia. Dev Change. 2012;43(6):1361-84.

7. Cordisco-Tsai L. Household Financial Management and Women's Experiences of Intimate Partner Violence in the Philippines: A Study Using Propensity Score Methods. Violence Against Women. 2017;23(3):330-50.

8. Beccaria G, Beccaria L, Dawson R, Gorman D, Harris J, Hossain D. Nursing Student's Perceptions and Understanding of Intimate Partner Violence. Nurse Education Today. 2013;(33):907-11.

9. Caetano P, Ísper A, Moreira R, Reatto D, Sabila C. Violencia contra la mujer: levantamiento de casos registrados en una Comisaría del Estado de Minas Gerais, Brasil. Rev Cienc Salud. 2017;15(2):203-210. doi:

10. Observatorio de Mujeres y Equidad de Género de la Secretaría Distrital de la Mujer. Información preliminar violencia física de pareja del Sistema de Información de Clínica y Odontología Forense, Instituto Nacional de Medicina Legal y Ciencias Forenses. Datos 2016 entregada a la SDMujer como respuesta al requerimiento 021-2017 del 23 de enero de 2017. 
11. Posada-Zapata I, Mendoza-Ríos A, Orozco-Giraldo I, Restrepo-Correa C, Cano-Bedoya S. Roles de género y salud en mujeres desplazadas por la violencia en Medellín, 2013-2014. Rev Cienc Salud. 2017;15(2):189-202. doi: 